\title{
Síndrome coronariana aguda em paciente jovem com sintomas atípicos
}

\author{
Acute coronary syndrome in young patients with atypical symptoms
}

\author{
Gustavo Daher ${ }^{a}$, Henrique Lane Staniak ${ }^{b}$, Márcio Sommer Bittencourt ${ }^{b}$ \\ Rodolfo Sharovskyc
}

Daher G, Staniak HL, Bittencourt MS, Sharovsky R. Síndrome coronariana aguda em paciente jovem com sintomas atípicos. Autopsy Case Rep [Internet]. 2011;1(1):63-7.

\section{RESUMO}

O diagnóstico da dor torácica aguda no setor de emergência pode ser difícil e desafiador, incluindo diversos diagnósticos diferenciais, dentre eles: causas benignas a causas potencialmente fatais. O diagnóstico na maioria das vezes é estabelecido através do quadro clínico e de exames complementares como: marcadores de necrose miocárdica, eletrocardiograma e radiografia de tórax. A angiotomografia coronariana tem sido utilizada nos casos de dor torácica aguda com baixa ou intermediária probabilidade de síndrome coronariana aguda, permitindo sua exclusão e conseqüente alta precoce dos pacientes. Apresentamos relato de caso de uma paciente jovem com dor precordial atípica e marcadores de necrose miocárdica discretamente elevados. Considerando-se a epidemiologia e o quadro clínico da paciente, foi feita hipótese diagnóstica inicial de miocardite. A angiotomografia cardíaca com pesquisa de realce tardio foi solicitada. Apesar do escore de cálcio ter sido zero, foi diagnosticada lesão estenosante grave no terço médio da artéria coronária descendente anterior com realce tardio na parede anterior do ventrículo esquerdo, compatível com necrose miocárdica. A paciente foi submetida a cateterismo com angioplastia da lesão.

Palavras-Chave: Dor no peito; Síndrome coronariana aguda; Tomografia; Angiografia/método; Miocardite.

\footnotetext{
a Residente na Divisão de Clínica Médica do Hospital Universitário - Universidade de São Paulo, São Paulo/SP - Brasil.

${ }^{b}$ Divisão de Clínica Médica do Hospital Universitário - Universidade de São Paulo, São Paulo/SP - Brasil.

C Serviço de Iconologia do Hospital Universitário - Universidade de São Paulo, São Paulo/SP - Brasil.
} 


\section{ABSTRACT}

Evaluation of acute chest pain can be extremely difficult and challenging. This diagnosis comprehends several causes, including benign and potentially lethal causes. Most of the time, the diagnosis can be done with the history and ordinary tests such as electrocardiogram, chest $x$-ray and myocardial enzymes. The coronary angiotomography is a new tool that can safely rule out acute coronary syndrome, being performed in patients with low to middle probability of acute coronary syndrome.

The present report describes a young woman that presented to the emergency unit with atypical chest pain and myocardial enzyme elevation. Miocarditis was the initial diagnosis

The patient was submited to a coronary angiotomography with late enhancement research. The coronary angiotomography demonstrated zero calcium score with a high degree stenosis in the coronary descending artery and late enhancement in anterior wall demonstrating myocardial necrosis. It was performed coronary catheterization and angioplasty afterwards.

Keywords: Chest paint; Acute coronary syndromes; Tomography; Angiography/methods; Myocarditis.

\section{INTRODUÇÃO}

A abordagem diagnóstica inicial de dores torácicas na unidade de emergência nem sempre é simples. Apresentações clínicas atípicas, sintomas mal caracterizados e pacientes que não sabem referir a história de forma adequada estão entre os diversos fatores que podem interferir com a definição clara do diagnóstico. A abordagem das dores torácicas inclui avaliação de sintomas clínicos, comorbidades, fatores de risco cardiovasculares, parâmetros do exame físico, eletrocardiograma e marcadores de necrose miocárdica, preferencialmente a troponina ${ }^{1}$. Mesmo com o uso destes dados, muitos pacientes permanecem sem um diagnóstico claro, e diversos exames complementares podem ser utilizados.

Dentre os principais diagnósticos diferenciais a serem avaliados no pronto socorro, as síndromes coronarianas agudas devem sempre ser lembradas. Para sua avaliação podem-se utilizar diversos exames, incluindo exames de estresse, como o teste ergométrico, e exames anatomicos, como a angiotomografia de artérias coronárias. Este é um exame recente na prática clinica, mas que demonstrou alta acurácia diagnóstica nestes casos $^{2}$.

Neste relato apresentamos o caso de um paciente do sexo feminino, jovem, tabagista que apresentava dor torácica atípica e que foi avaliada com angiotomografia de artérias coronárias.

\section{Relato do caso}

Mulher de 33 anos de idade, tabagista, sem antecedentes cardiovasculares, procurou o pronto socorro com dor precordial atípica, reprodutível à palpação de musculatura paravertebral, irradiando para dorso associada a dispnéia aos esforços há 3 dias. No momento da entrada encontrava-se assintomática em repouso. Os exames laboratoriais da admissão são exibidos na Tabela 1.

Tabela 1 - Exames laboratoriais durante a internação.

\begin{tabular}{ll}
\hline Exame & Dia da admissão \\
\hline Glicose & $91 \mathrm{mg} / \mathrm{dl}$ \\
Uréia & $26 \mathrm{mg} / \mathrm{dl}$ \\
Creatinina & $0,8 \mathrm{mg} / \mathrm{dl}$ \\
Potássio & $4,2 \mathrm{mg} / \mathrm{dl}$ \\
Colesterol Total & $212 \mathrm{mg} / \mathrm{dl}$ \\
HDL & $43 \mathrm{mg} / \mathrm{dl}$ \\
LDL & $134 \mathrm{mg} / \mathrm{dl}$ \\
Triglicérides & $177 \mathrm{mg} / \mathrm{dl}$ \\
\hline
\end{tabular}


Exame clínico inicial com pressão arterial de $140 / 90 \mathrm{mmHg}$ em ambos os membros superiores, freqüência cardíaca de 80 bpm, sem alteração de pulsos nos quatro membros. Peso $60 \mathrm{~kg}$, altura $1,53 \mathrm{~m}$ e IMC de 25,6 . Ausculta cardíaca e pulmonar sem alterações.
Foram solicitados marcadores de necrose miocárdica e eletrocardiograma. A primeira dosagem de marcadores de necrose demonstrou resultado acima do limite superior (Tabela 2)

Tabela 2 - Curva de marcadores de necrose miocárdica (dias da admissão).

\begin{tabular}{lccc} 
& $1^{\circ}$ & $2^{\circ}$ & $3^{\circ}$ \\
\hline CKMB & $24,8 \mathrm{ng} / \mathrm{dl}$ & $116 \mathrm{ng} / \mathrm{dl}$ & $6,9 \mathrm{ng} / \mathrm{dl}$ \\
Troponina & $3 \mathrm{ng} / \mathrm{dl}$ & $35,9 \mathrm{ng} / \mathrm{dl}$ & 1,65 \\
\hline
\end{tabular}

O eletrocardiograma inicial demonstrou alterações inespecíficas da repolarização ventricular (Figura 1).

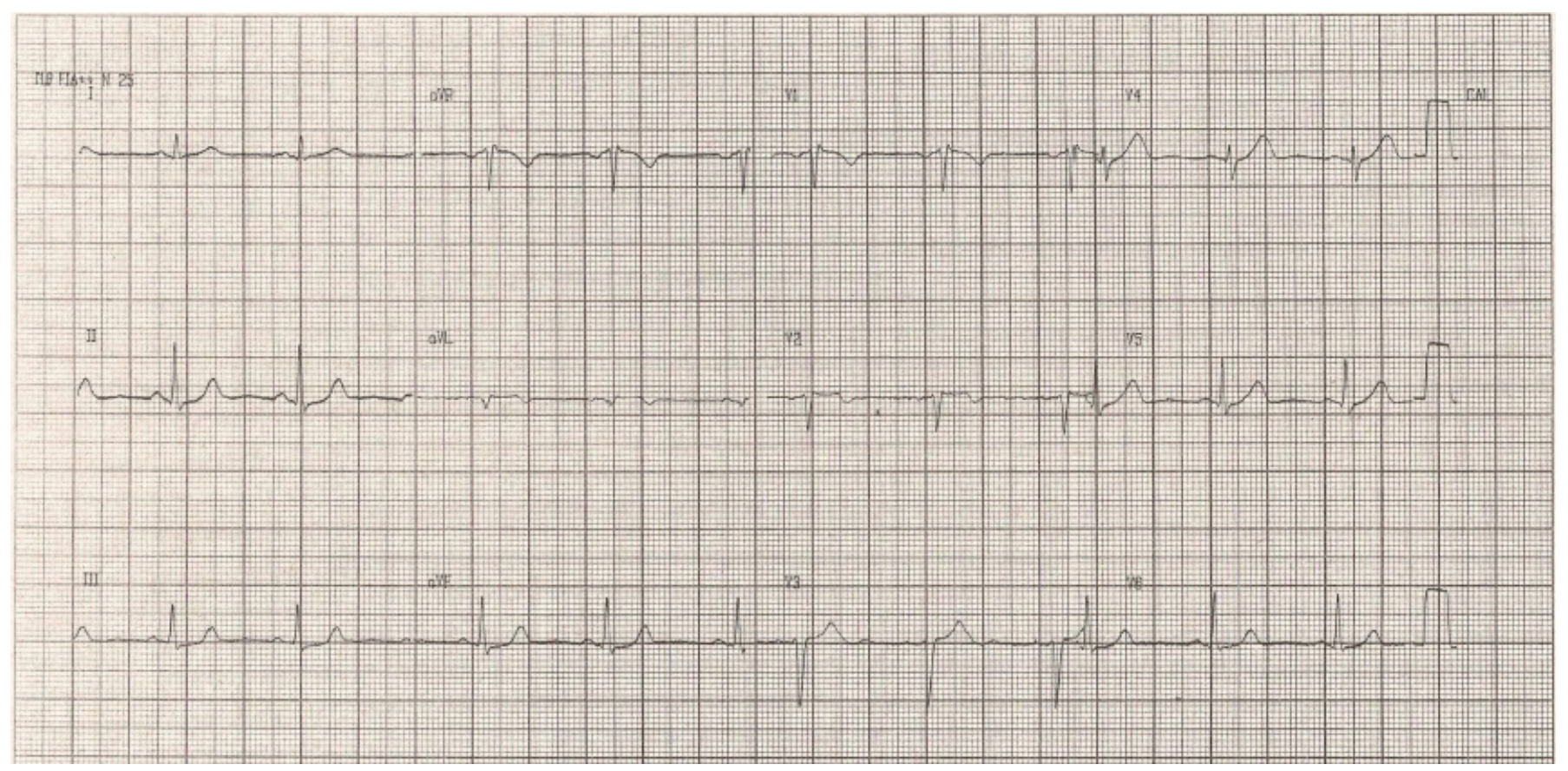

Figura 1 - Eletrocardiograma com ausência da progressão de onda R nas derivações V1 a V3 e alteração difusa da repolarização ventricular.

O ecocardiograma demonstrou comprometimento segmentar do miocárdio de grau discreto em parede antero septal médio e apical com fração de ejeção de 45\%. Átrio esquerdo de $38 \mathrm{~mm}$, septo interventricular e parede posterior de $9 \mathrm{~mm}$ e demais câmaras cardíacas de dimensões normais. Considerando a paciente como de baixo risco cardiovascular, com dor atípica, sintomas e ecocardiograma compatíves com insuficiência cardíaca, foi considerado o diagnóstico diferencial de miocardite e síndrome coronariana aguda como causas da dor torácica e alteração de marcadores de necrose miocardica. Desta forma foi solicitada angiotomografia coronariana para avaliação de doença coronária e pesquisa de realce tardio associado à miocardite.

A angiotomografia cardíaca foi realizada de forma prospectiva, com aquisição de imagem sem contraste para avaliação do escore de cálcio, imagem contrastada para visualização das artérias coronárias e nova imagem tardia para pesquisa do realce tardio. A dose total de radiação foi de $5,5 \mathrm{mSv}$. O escore de cálcio da paciente foi zero, a angiotomografia demonstrou estenose grave por placa não calcificada em terço médio de artéria descendente anterior (Figura 2B). 


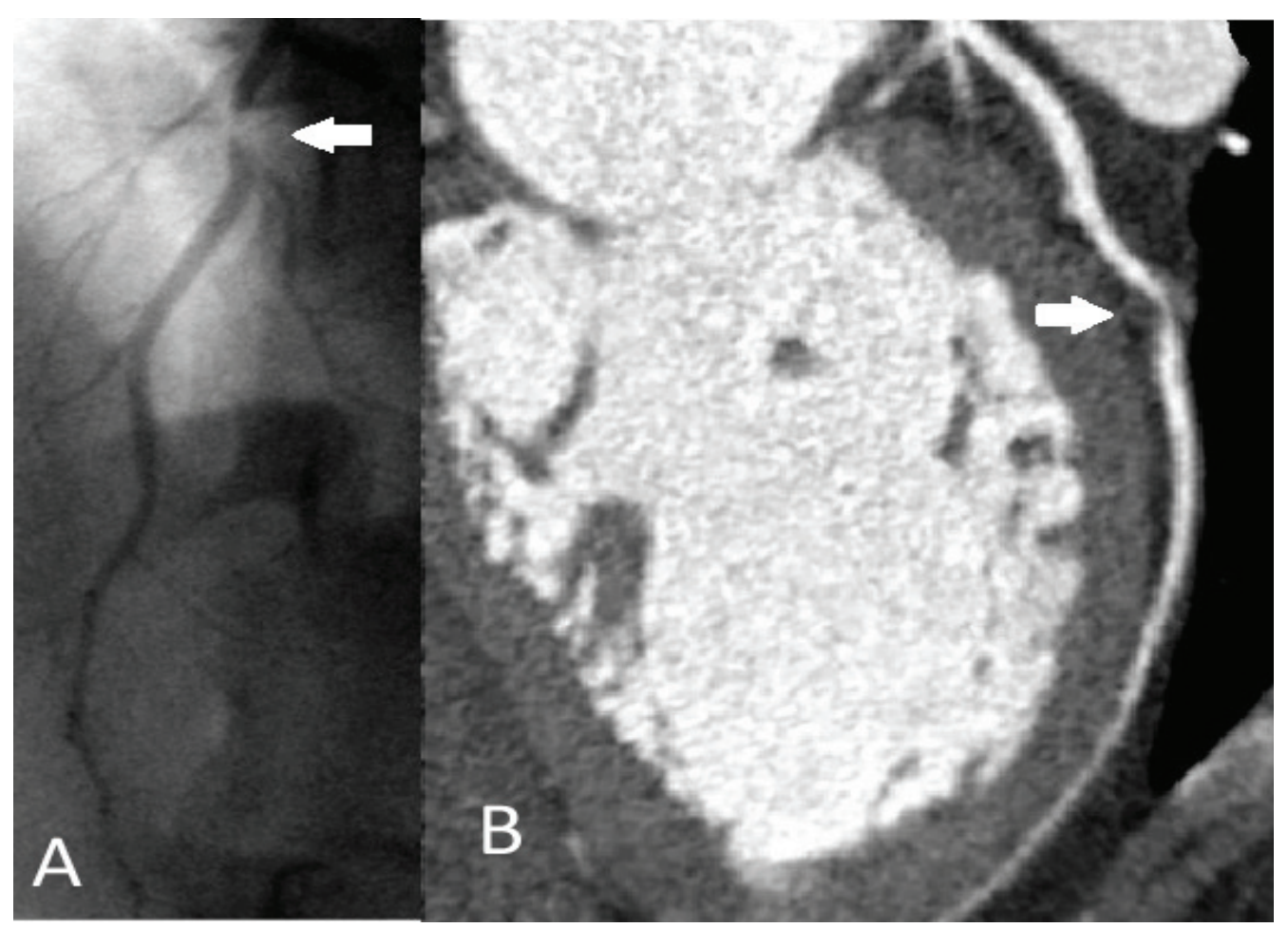

Figura 2A - Imagem da coronariografia invasiva de artéria descendente anterior, com lesão grave em seu terço proximal (seta). B: Reconstrução multiplanar de artéria descendente anterior. Seta: Placa não calcificada com obstrução luminal > $70 \%$ em terço médio de artéria descendente anterior esquerda.

As outras artérias não apresentavam alterações significativas. A pesquisa de realce tardio demonstrou realce segmentar em região antero septal, compátivel com realce causado pelo quadro de infarto agudo do miocárdio (Figura 3).

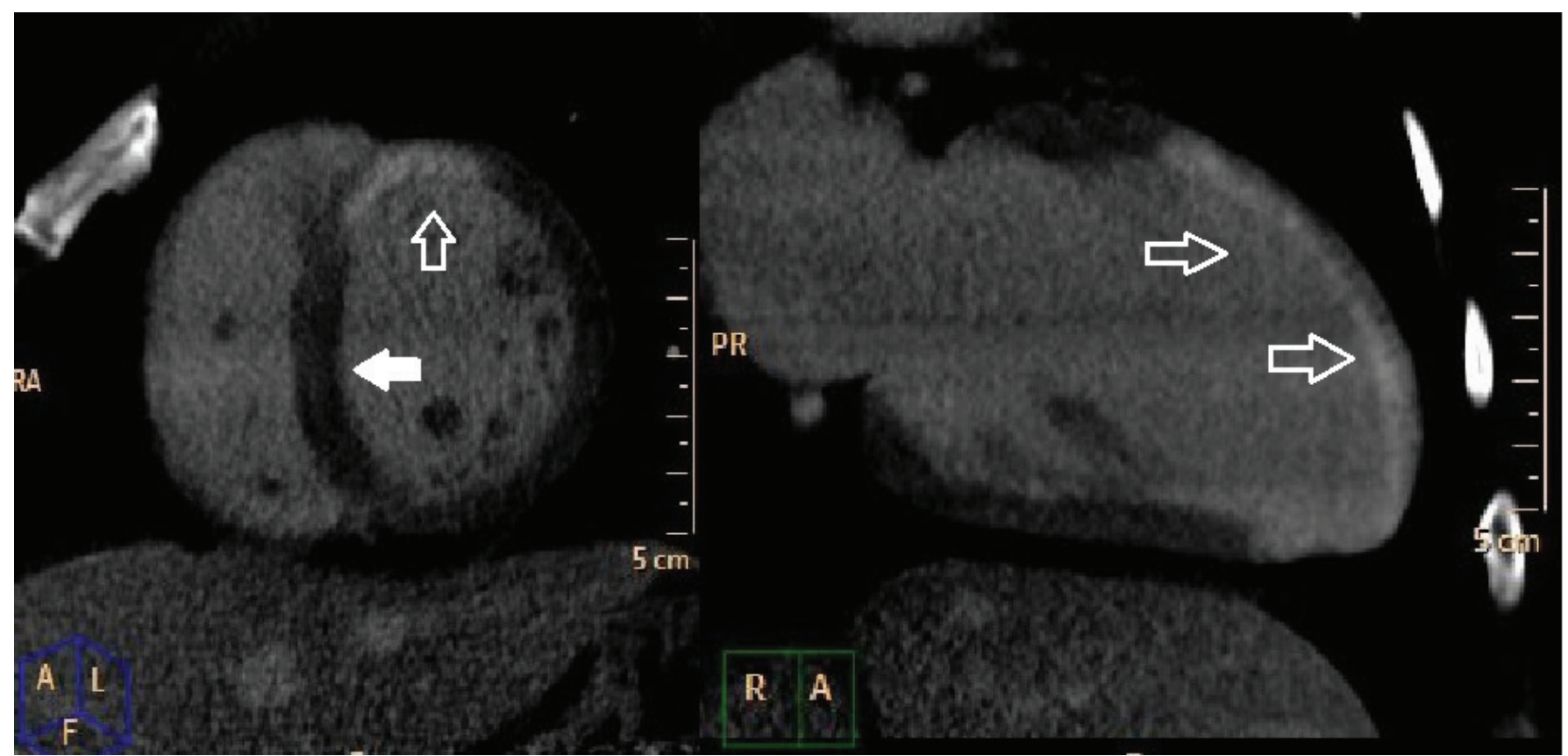

Figura 3A - Eixo curto do coração em terço médio entra a valva mitral e o ápice cardíaco, com presença de realce tardio em parede anterior e Antero-septal de porção média do ventrículo esquerdo. (seta não preenchida). A seta preenchida demonstra atenuação miocárdica normal. B - Eixo longo do coração demonstrando realce tardio de parede anterior e septal de ventrículo esquerdo (setas vazadas). 
Foi feito o diagnóstico de síndrome coronariana aguda sem supradesnivelamento do segmento ST e iniciadas medidas clínicas para o seu tratamento. A paciente foi encaminhada para cateterismo cardíaco, evidenciando obstrução em terço médio de artéria descendente anterior de $80 \%$ (Figura $2 A$ ), sendo realizada angioplastia com stent não farmacológico.

A paciente evoluiu com melhora clínica e permaneceu assintomática recebendo alta para seguimento ambulatorial.

\section{DISCUSSÃO}

A melhor indicação de angiotomografia de artérias coronárias para a avaliação de dor torácica em pronto socorro é para paciente de baixo ou intermediário risco com eletrocardiograma normal e marcadores de necrose sem alterações. A paciente do caso descrito era de baixo risco, e por isso sua alteração de marcadores foi considerada como uma possível miocardite. Neste caso, a angiotomografia foi indicada para descartar doença coronária. Alguns relatos têm demonstrado seu potencial uso para a avaliação de realce tardio, uma alteração

\section{REFERÊNCIAS}

1. 1. Anderson JL, Adams CD, Antman EM, et al. ACC/ AHA 2007 guidelines for the management of patients with unstable angina/non-ST-elevation myocardial infarction: a report of the American College of Cardiology/American Heart Association Task Force on Practice Guidelines. Circulation. 2007;116(7):e148-e304.

2. Hoffmann U, Bamberg F, Chae CU, et al. Coronary computed tomography angiography for early triage of patients with acute chest pain: the ROMICAT (Rule Out Myocardial Infarction using Computer Assisted Tomography) trial. J Am Coll Cardiol. 2009;53(18):1642-50.

3. Redheuil AB, Azarine A, Garrigoux P, Mousseaux E. Cor- típica de pacientes com miocardite ${ }^{3}$. Apesar do uso não rotineiro desta técnica, a indicação neste caso permitiu avaliar adequadamente as duas hipóteses diagnósticas.

Desta forma, foi diagnosticada lesão grave que permitiu a indicação de intervenção coronariana percutânea. Além disso, a imagem de realce tardio demonstrou a extensão da área do infarto.

Outra particularidade importante deste exame é que a paciente apresentava escore de cálcio zero, apresar de lesão coronária grave. Nos pacientes assintomáticos o escore de cálcio esta diretamente relacionado com doença coronária e eventos futuros ${ }^{4}$. No entanto, em pacientes com quadros agudos, mesmo o escore de cálcio zero é incapaz de descartar doença coronária ${ }^{5}$, como se demonstrou nesta paciente.

Em conclusão, a angiotomografia de artérias coronárias é um exame útil na avaliação diagnóstica de pacientes com baixa ou intermediária probabilidade de doença coronária, que apresentem dor torácica de etiologia não definida. respondence between delayed enhancement patterns in multislice computed tomography and magnetic resonance imaging in a case of acute myocarditis. Circulation. 2006;114(19):e571-2.

4. Detrano R, Guerci AD, Carr JJ, et al. Coronary calcium as a predictor of coronary events in four racial or ethnic groups. New Engl J Med. 2008;358(13):1336-45.

5. Fernandez-Friera L, Garcia-Alvarez A, BagheriannejadEsfahani $\mathrm{F}$, et al. Diagnostic value of coronary artery calcium scoring in low-intermediate risk patients evaluated in the emergency department for acute coronary syndrome. Am J Cardiol. 2011;107(1):17-23.

\section{Conflito de interesse: Não}

Submetido em: 08 de Fevereiro de 2011

Aceito em: 28 de Fevereiro de 2011

Correspondência: Divisão de Clínica Médica

Av. Prof. Lineu Prestes 2565 - Cidade Universitária - USP - São Paulo - Brasil

CEP 05508-900

E-mail: msbittencourt@bol.com.br 